\title{
Flow effects on the morphology and dynamics of aeolian and subaqueous barchan dunes
}

\author{
Pascal Hersen \\ Laboratoire de Physique Statistique, Ecole Normale Supérieure, Paris, France
}

Physics Department, Danish Technical University, Lyngby, Denmark

Received 15 June 2004; revised 19 April 2005; accepted 18 May 2005; published 16 November 2005.

[1] Barchans are aeolian sand dunes that form where unidirectional winds blow on firm ground with limited sand supply. Although they have been analyzed for decades, the difficulty of conducting experiments in the field, due to the large lengthscale and timescale involved, is a considerable obstacle to understand their dynamics. However, crescentic patterns also appear underwater on a much smaller scale, allowing easier study. It is shown here that down-scaled solitary barchans can be created in the laboratory using the periodic and asymmetric motion of a plate underwater to simulate the effect of a unidirectional flow. Those subaqueous barchans compare very well to their aeolian relatives. Their speeds scale as the cube of the speed of the flow times the inverse of their typical dimension. Finally, the exploration of new topics, such as the influence of the directionality of the wind or that of binary collisions, illustrate that barchans can eventually adapt to any external perturbations by emitting one or several small barchans.

Citation: Hersen, P. (2005), Flow effects on the morphology and dynamics of aeolian and subaqueous barchan dunes, J. Geophys. Res., 110, F04S07, doi:10.1029/2004JF000185.

\section{A Short Introduction to Barchan Dunes}

[2] The shapes of aeolian sand dunes originate from their interaction with the wind. In fact, the wind can shape dunes by way of erosion and deposition processes, but on the other hand, dunes are large enough to modify the velocity profile of the wind. A century of field observations has demonstrated that the shape of aeolian dunes is mainly determined by the directionality of the wind and sand availability [Bagnold, 1941; Cooke et al., 1993]. When the wind is unidirectional and the sand supply is low, flat, crescentic dunes, called barchans, appear and migrate along the wind direction.

\subsection{Aeolian Barchan Dunes: Basics}

[3] A typical barchan (see Figure 1) has a flat windward side $\left(\sim 10^{\circ}\right)$ whereas the lee-side, usually called the slip-face and formed by successive avalanches, is steeper $\left(\sim 34^{\circ}\right)$. On its lateral sides, two horns extend along the wind direction, forming the typical crescentic shape and letting some sand escape from the barchan. The width and length of barchans range between $15 \mathrm{~m}$ and $150 \mathrm{~m}$, while the height ranges from $1 \mathrm{~m}$ to $15 \mathrm{~m}$. Furthermore, no mature barchan smaller than typically $15 \mathrm{~m}$ long and $1 \mathrm{~m}$ high develops in the desert [Bagnold, 1941; Andreotti et al., 2002]. For barchans large compared to this critical size, the height, $H$, the width, $W$, and the length, $L$, are almost linearly dependent and therefore, barchans can be considered as self-similar shapes [Sauermann et al., 2000].

Copyright 2005 by the American Geophysical Union. 0148-0227/05/2004JF000185\$09.00
[4] Barchans are also known for their high mobility. This comes from erosion and deposition processes: the wind carries sand grains from the bottom of the windward side up to the crest. There they are deposited because of the dramatic decrease of the velocity of the wind just after the crest. When the upper part of the slip-face becomes too steep, an avalanche spontaneously nucleates and redistributes the sand along its side, while the slope of the slip-face relaxes toward its equilibrium value. Thereby grains pass from the back of the dune to the slip-face and the dune moves. Field surveys have shown that the velocity of barchan dunes, $c$, varies with the size of dunes as $c \simeq Q /\left(H+H_{0}\right)$ [Cooke et al., 1993; Andreotti et al., 2002], where $Q$ has the dimension of a volumic sand flux and varies with the strength of the wind [Hastenrath, 1967, 1987], and $H_{0}$ is a cutoff length which takes into account the fact that very small dunes cannot move faster than the wind.

[5] However, hardly anything more is known concerning the effect of the flow on the shape and on the dynamics of barchan dunes. This is due to the difficulty of achieving precise measurements in the field, because of the large lengthscale and timescale involved and the lack of control on the wind. Therefore it is crucial to investigate this issue under controlled conditions in the laboratory. However, the existence of a minimal size for barchan dunes means that no barchan can be reproduced in air at the laboratory scale [Bagnold, 1941; Andreotti et al., 2002; Dauchot et al., 2002]. This critical size comes from the inertia of sand grains. Let us consider the simple situation in which a wind, that carries no grain, arrives on a flat sand bed. Grains are progressively set into motion by the wind, and the sand flux increases with the distance from the beginning of the sand 


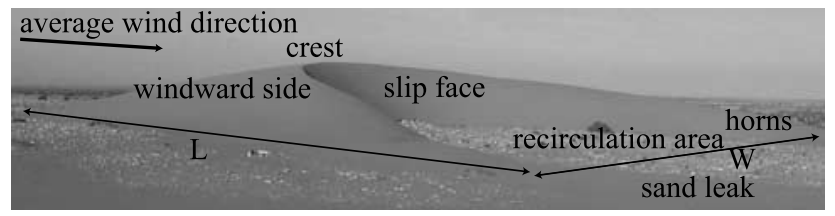

Figure 1. Morphology of a barchan dune from the region of Tarfaya, southwestern Morocco. Its length is approximately $30 \mathrm{~m}$. The typical morphological elements of a barchan are visible: a flat windward side, a steep slip-face, and two horns pointing in the wind direction and letting some sand escape from the dune. When this sand leak is compensated by an incoming sand flux, the barchan migrates shape-invariantly downwind. The air flow separates at the crest leading to the formation of a recirculation flow in the sheltered part, between the horns.

bed. Simultaneously, the moving grains absorb momentum from the wind and accordingly the wind slows down until on average no new grains can be put into motion. Therefore, after some transient, the sand flux is saturated. This transient induces a space lag between the sand flux $q$ and its value in the saturated regime, $q_{s}$ [Anderson et al., 1991; Sauermann, 2001; Kroy, 2002; Andreotti et al., 2002; Valance, 2005]. This saturation length, $l_{s}$, is the characteristic lengthscale over which the sand flux adapts to a change of the wind velocity or the sand supply. For a sand pile smaller than a few times $l_{s}$, the sand flux increases everywhere on its surface, and accordingly the sand pile is just swept away. On the contrary for a longer sand pile, the sand flux can become over-saturated and deposit sand in its lee side: the sand pile can transform into a propagating sand dune [Andreotti et al., 2002; Kroy, 2002]. It has been shown that this saturation length varies as the inertia length, $l_{\text {drag }}$ [Andreotti et al., 2002; Valance and Langlois, 2005; Valance, 2005] which is the typical length needed for a sand grain to reach the velocity of a turbulent flow. This length can be expressed as

$$
l_{\text {drag }}=\frac{\rho_{s}}{\rho_{f}} d \sim 0.5 \mathrm{~m}
$$

in which $\rho_{s} \sim 2500 \mathrm{~kg} \mathrm{~m}^{-3}$ is the sand density, $d \sim 200 \mu \mathrm{m}$ is the typical diameter of sand grains and $\rho_{f} \sim 1 \mathrm{~kg} \mathrm{~m}^{-3}$ is the density of air. This strongly suggests that increasing the density of the fluid, $\rho_{f}$ reduces the saturation length and accordingly the minimal size, leading to small-scale barchan dunes [Hersen et al., 2002].

\subsection{Subaqueous Barchans Ripples as a Model of Aeolian Barchans}

[6] In particular, crescentic ripples of tens of centimeters are often observed in rivers or marine environment [Allen, 1968; Mantz, 1978]. The origin of their formation lies in the same underlying physics as for aeolian dunes: a unidirectional current that shapes the sand bed and interacts with it. However, in water, $l_{\text {drag }}$ is approximately one thousand times smaller than in air, and therefore crescentic ripples are expected to compare well to aeolian barchans first rescaled by $l_{\text {drag. }}$. However, the subaqueous crescentic ripples usually appear in areas with a large sand supply. This is the reason why one generally observes many crescentic structures merging together and forming complex three dimensional ridges that propagate along the current direction. This has been nicely shown by the experiments of Mantz [1978]: A flat and uniform sand bed submitted to a uniform water current gives rise to many crescentic ripples that grow and merge as long as there is sand between the different structures. As a result, studies of subaqueous solitary barchans are very rare [Best, 2004]. Nevertheless, this does not preclude the possibility of observing solitary crescentic dunes under water providing that the sand supply is dramatically lowered. This is why we have designed a simple experiment to reproduce solitary underwater barchans from a single conical sand pile rather than a uniform sand bed. This study should be significant not only for the understanding of aeolian barchans, since it will provide us with new insights on their dynamics, but also for shedding light on marine sand wave dynamics since barchans can be seen as the elementary structure forming the more complex barchanoid ridges that are usually observed in river environment.

\section{Studying Barchans in the Laboratory}

[7] The usual method to reproduce unidirectional flows is to make use of circulating water in a water flume driven by a strong water pump. Although this method is relevant, its utilization is restrained by the strength of the pump. Here the cross section of the flume must be large enough to avoid both border effects and free surface interactions with the sand bed. These two requirements are moreover constrained by the average speed of the flow which should exceed the threshold speed of motion for sand grains. Therefore high discharges are usually required. Under water, and for grains of $150 \mu \mathrm{m}$, this critical velocity is roughly given by: $u_{*_{c}}=0.5 \mathrm{~cm} \mathrm{~s}^{-1}$ [Julien, 1998]. Taking a flume $0.4 \mathrm{~m}$ wide and imposing a water depth of $0.1 \mathrm{~m}$, the discharge is of the order of $\sim 4 \mathrm{~L} \mathrm{~s}^{-1}$ [Julien, 1998]. Entraining grains with a velocity of the flow far from the threshold necessitates an even larger water discharge. Such high discharges require specially designed pumps and are far from the tabletop experiment we want to describe here.

\subsection{Description of the Experimental Setup}

[8] A neat alternative, inspired by laboratory experiments conducted on vortex ripples [Hansen et al., 2001], consists on moving a plate under water: in the moving frame the sand feels a relative water current whose speed is the opposite of that of the plate. On that basis, it is very easy to exceed the threshold of motion of sand grains. More precisely, in a water-tank filled with water (see Figure 2), a conical pile made with glass beads of $150 \mu \mathrm{m}$ is formed on a plate. This plate is then oscillated asymmetrically at the constant depth of $0.12 \mathrm{~m}$ by the way of an external motor. First, the plate is accelerated during a very short time $t_{a}=$ $0.150 \mathrm{~s}$ to reach a constant speed velocity $v=0.28 \mathrm{~m} \mathrm{~s}^{-1}$. The plate moves at this velocity during $t_{b} \sim 1 \mathrm{~s}$ and then decelerates during the time $t_{c}=1 \mathrm{~s}$ and finally stops smoothly. This deceleration has to be slow enough to 


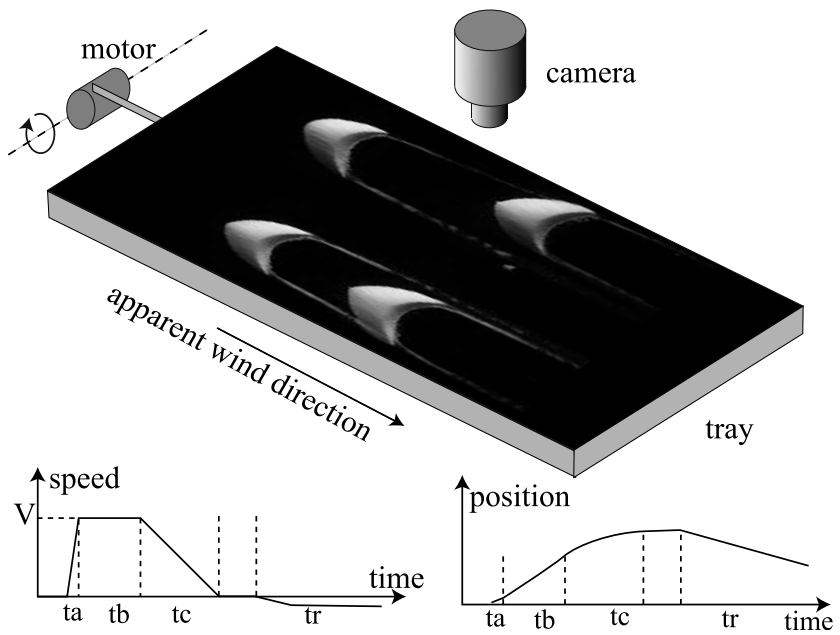

Figure 2. Experimental setup. The dimensions of the plate are $40 \times 30 \mathrm{~cm}$, and the amplitude of one oscillation of the plate is $50 \mathrm{~cm}$. The shape of the sand pile is monitored by a CCD video camera above the plate and moving with it. The picture shows four subaqueous barchans, approximately $5 \mathrm{~cm}$ wide, that have been created with this experiment. This shows its ability to easily produce one or several barchan dunes. Notice the sand leak developing from the horns along the direction of the wind.

prevent any strong counter flow to occur. In practice, the counter flows do not change the morphology of barchans but rather slightly smooth the crest line. Using the decelerating time of $1 \mathrm{~s}$ allows to diminish the effect of this drawback and, at any rate, the sharpness of the crest will be recovered almost instantaneously during the next oscillation. Finally, after a stop of $\sim 1 \mathrm{~s}$, the plate slowly comes back to its starting position so no grain can move. This back and forth motion with a total period of approximately $20 \mathrm{~s}$ is then repeated several times, while a video camera monitors the evolution of the shape of the sand pile. This particular motion is thereafter called the reference motion.

\subsection{Formation and Propagation of Subaqueous Barchans}

[9] Using the asymmetric motion described above, an initial conical sand pile (6.5 g of $150 \mu \mathrm{m}$ glass beads) transforms into a propagating crescentic shape. The initial conical shape is not adapted to the water flow: a strong erosion occurs on the "windward" side and the flow separates at the summit. This is why, after a few strokes of the plate, the slip-face is fully formed. Then two horns grow on the lateral side of the sand pile (see Figure 3). Finally when the sand pile has moved approximately over its own length, the crescentic shape has formed and propagates downwind with a constant velocity as seen in Figure 3. Apart from the obvious morphological resemblance with aeolian barchans, other similarities are to be noticed. First there is a flow separation at the crest and accordingly a recirculation area in the lee of the dune. Second, two narrow lines of sand develop from the tip of the horns in the direction of the wind. This sand leak is also observed for aeolian barchans. This means that the subaqueous barchans actually shrink while propagating, since there is no incoming sand flux in the experiment. However, the loss is so small that the barchan, to a good approximation, can be considered in a steady state, as can be observed in Figure 3. Finally, notice that starting from a flat sand bed, many barchans of different sizes appear and propagate as in the case of the water-flume experiment of Mantz [1978]. This suggests that this experiment is able to simulate correctly the effect of a unidirectional current on a bedform.

\subsection{Morphological Comparison With Aeolian Barchans}

[10] Varying the mass of the initial conical sand pile, barchans of variable sizes have been made in order to compare their morphology to that of "real" aeolian barchans. This can be done by rescaling the dimensions of barchans by $l_{\text {drag. }}$. As a matter of fact, under water and with grains of $150 \mu \mathrm{m}$ the inertia length is now very small: $l_{\text {drag }} \simeq 0.375 \mathrm{~mm}$. Figure 4 shows a rather good agreement between the dimensions of underwater barchans and that of aeolian barchans once rescaled by $l_{\text {drag }}$. Notice that in both cases no barchans smaller than typically $20 l_{\text {drag }}$ have been observed, confirming the existence of a minimal size. These results are strong evidence for both the legitimacy of this experimental method and the physical relevance of $l_{\text {drag. }}$. In other words, it confirms the direct link between large scale aeolian patterns and small scale underwater ripples made with this experiment. It should be noted that this is a very important result since the motion of sand grains are not the same under water (creeping motion and direct transportation) and in air (saltation and reptation) [Bagnold, 1941; Anderson et al., 1991; Julien, 1998]. This paradox shows that the only lengthscale of this problem comes from the saturation length of the sand flux, and not from the specific trajectories followed by sand grains.

\subsection{Dynamical Comparison With Aeolian Barchans}

[11] To achieve the comparison with aeolian barchans it is also imperative to investigate the dynamics of subaqueous barchans. Figure 5 shows that the velocity scales as $c \sim m^{-1 / 3}$ where $m$ is the initial mass of the barchan. As their width scales like $m^{1 / 3}$ (see inset of Figure 5) and the width, the

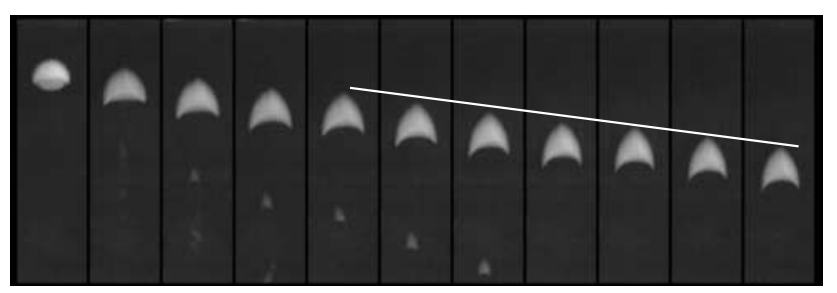

Figure 3. Formation and evolution of a subaqueous barchan dune created by the experimental setup. Starting from an initial conical sand pile ( $\sim 4 \mathrm{~cm}$ wide), a barchan dune quickly forms and propagates. The sand pile rapidly adapts to the water stream. The emergence of a slip-face is accompanied by the nucleation of a small barchan dune downwind. However, after some transient, horns are well developed and the classic shape of a crescent is recovered. The straight line and the direct observations of the shape show that even if there is a small sand leak from the tip of the horns, the dune evolves quasi-steadily. There are 10 strokes of the plate between each picture. 
rescaled height $\mathrm{H} / 1_{\mathrm{drag}}$

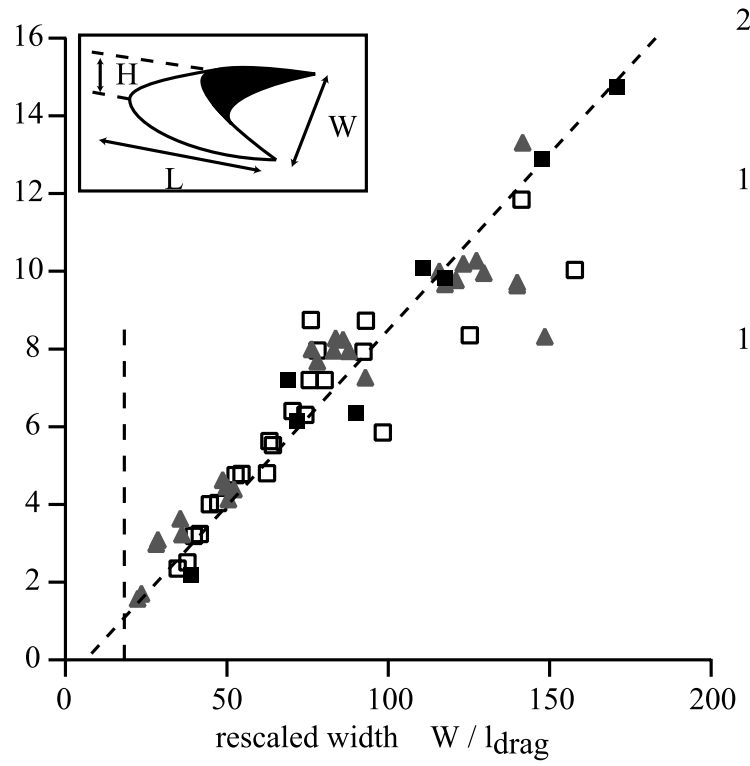

rescaled length $\quad \mathrm{L} / 1_{\mathrm{drag}}$

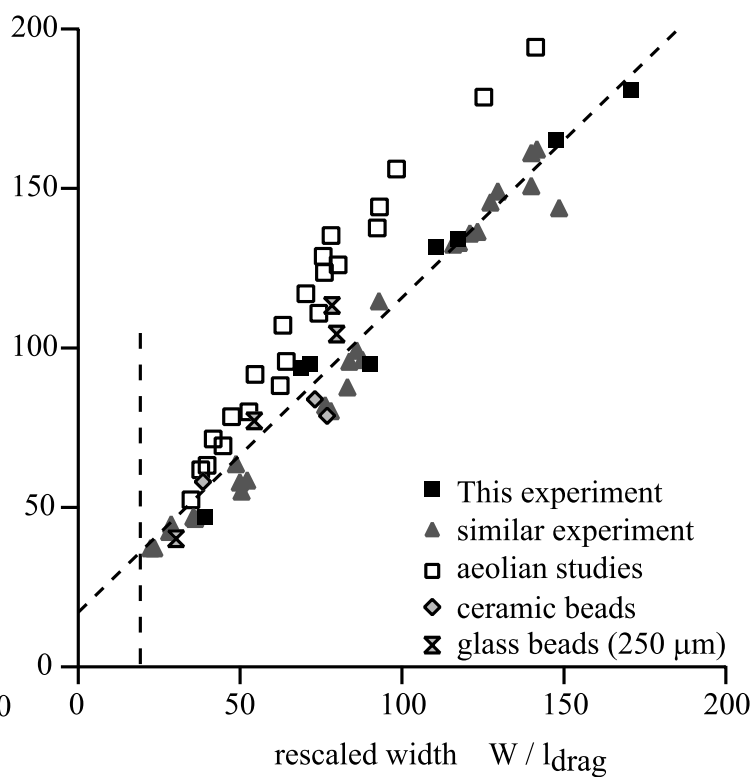

Figure 4. Morphological comparison between aeolian and subaqueous barchans. The width, the length, and the height match very well once rescaled by $l_{\text {drag. }}$. The small difference of the ratios $L / W$ between aeolian and subaqueous dunes could come from the difficulty to measure precisely the length on the field. The height is estimated by measuring the length of the slip-face and using the fact that for glass beads this slip-face always makes an angle of $\simeq 24^{\circ}$ to the horizontal. The results of this experiment $(\sim 150 \mu \mathrm{m})$ are also in excellent agreement with a previous draft version of this experiment [Hersen et al., 2002], plotted here as shaded triangles, and where the height was measured using the deviation of a laser-line by the subaqueous dune. Finally, notice that using ceramic beads (density of 3, 7, and $80 \mu \mathrm{m}$ of diameter) as well as larger glass beads (diameter of $\sim 250 \mu \mathrm{m}$ ) also gives barchan dunes with the same aspect ratio. This is strong evidence for the relevance of both $l_{\text {drag }}$ and this experimental setup.

length and the height are linearly related, at least for large barchans, this leads to the conclusion that barchan dunes move inversely proportionally to their typical dimensions (either $W, L$ or $H$ ). This is in keeping with what has been found in the case of aeolian barchans. However, the speed of the subaqueous barchans is considerably larger than that of aeolian dunes. Typically a 5-cm-wide barchan migrates at the speed of $0.004 \mathrm{~cm} \mathrm{~s}^{-1} \simeq 1.2 \mathrm{~km} / \mathrm{yr}$ so its turnover time is approximately half an hour while the corresponding dune in air, around $50 \mathrm{~m}$ long, propagates approximately at $10 \mathrm{~m} / \mathrm{yr}$ [Cooke et al., 1993] and needs 5 years to migrate one wavelength.

[12] As a first conclusion, these measurements prove that this apparatus is an excellent alternative to investigate the dynamics of solitary barchan dunes in the laboratory. Typical timescales of the dynamics of barchans are shortened and the small sizes of the subaqueous structures allow very simple and precise monitoring. However, before looking at complex situations, it is worthwhile to bring into focus two points. First, it is now possible to investigate the relation between the velocity of the dune and that of the water flow. Second, it may be interesting to discuss the role of the acceleration process.

\section{Influence of the Flow Properties}

\subsection{Theoretical Derivation}

[13] Since the main objective of this article is to understand the influence of the velocity of the wind on the dynamics of barchans, it is interesting to clarify theoretically the expected scaling for $c$ in the case of aeolian dunes. This can be done in the simple case of a two dimensional dune migrating shape-invariantly at the velocity $c$. Calling $q$ the vertically integrated sand flux at the position $x$ and $h$ the local elevation, the equation of mass conservation is

$$
-c \partial_{x} h+\partial_{x} q=0
$$

A direct integration gives $q(x)=q_{0}+\operatorname{ch}(x)$. Applying this equation at the crest of the dune, we get: $c=q_{c} / H$. Therefore, if $q_{c}$ does not depend on the size of the dune, this simple derivation is in good agreement with field results. Actually, for a turbulent flow (for aeolian dunes one generally has $R_{e} \geq 10^{6}$ ) the velocity of the wind is known to be scale invariant. In other words, the velocity of the wind at the crest is the same for dunes of different sizes (apart from logarithmic scale factor) provided that they are selfsimilar [Sauermann, 2001]. As said previously, this is the case for large barchans and thus the shear velocity, $u_{*}$, is expected to depend only on dimensionless properties of the dune. This has led Sauermann [2001] to express the velocity of the wind on the back of the dune as

$$
u *(x)=u *, \infty(1+\mathcal{F}) .
$$

Here $\mathcal{F}\left\{H / L, \partial_{x} h\right\}$ is a scale-invariant functional of the dimensionless shape of the dune only and does not depend on its size. (Recent models use the following functional 


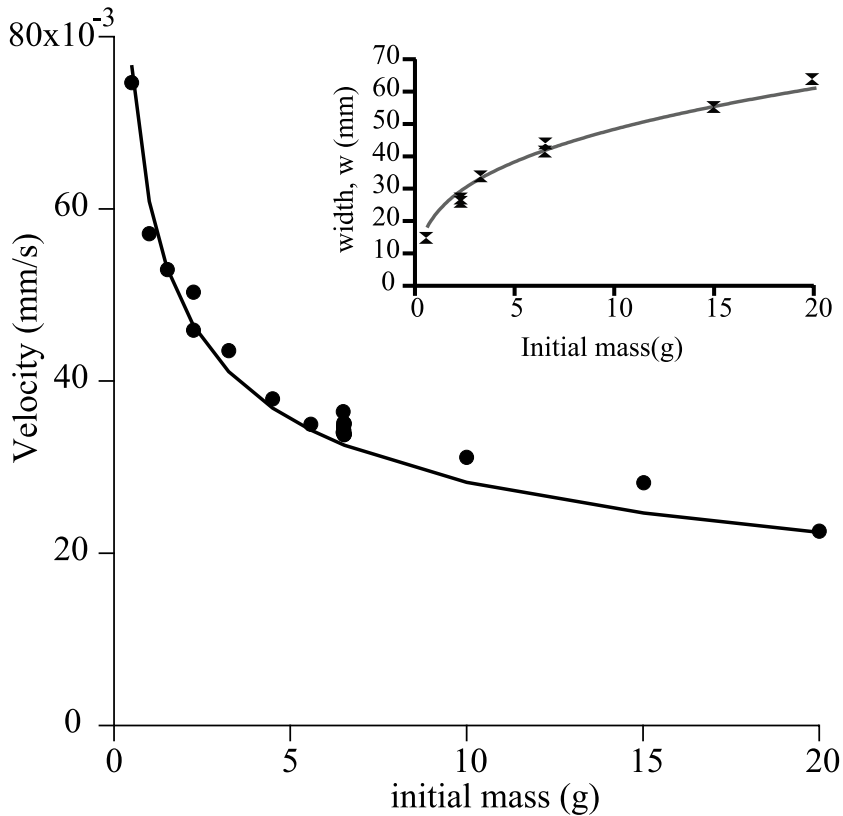

Figure 5. Migration rate of subaqueous barchans. Measuring the speed of barchans as a function of the mass shows that $c \sim \mathrm{m}^{-1 / 3}$ (black line). Furthermore, the inset shows that $W$ scales as $\mathrm{m}^{1 / 3}$, which is in agreement with the fact that barchans are scale invariant. This leads to $c \sim \mathrm{W}^{-1}$ as for aeolian barchans since the width, the height, and the length are linearly dependent. The speeds are expressed in real time.

form: $\mathcal{F}=A \int \frac{\partial_{x} h}{x-x^{\prime}} d x^{\prime}+B \partial_{x} h$ in which $A$ and $B$ are two phenomenological parameters. These models results are in good agreement with field observations.) Experimental investigations [Andreotti et al., 2002] show that the speedup at the crest $u_{*} / u_{*, \infty}$ is of the order of $1.3-1.4$ and does not depend much of the height of the dune, supporting the previous derivation. Moreover, it is well known that the saturated sand flux, $q_{s}$, is proportional to the cube of the shear velocity, has proposed first by Bagnold [1941],

$$
q_{s}=\frac{B(d) \rho_{s}}{\rho_{f} g} u_{*}^{3}
$$

in which $B(d)$ is a function of the size of the grain. This expression only holds for "strong" winds. For gentle winds, the strength of the wind acting on a grain $\left(\rho_{f} u_{*}^{2} d^{2}\right)$ is not sufficient to counterbalance its weight $\left(\rho_{s} g d^{3}\right)$, and no grains can move. Lettau and Lettau [1969] have proposed a slightly modified expression so as to take into account this threshold:

$$
\begin{gathered}
q_{s}=\frac{B(d) \rho_{f}}{\rho_{s} g}(u *-u *, c) u_{*}^{2}, u *>u *, c \\
q_{s}=0, u *<u *, c .
\end{gathered}
$$

Finally, since the dune is rather flat, it is possible to linearize equation (5) by using equation (3) to get

$$
q_{s} \sim u_{*, \infty}^{2}\left(u_{*, \infty}-u_{*}^{\prime}\right)(1+3 \mathcal{F})
$$

Here $u_{*}^{\prime}=u_{*, c}(1-\mathcal{F})$. Notice that the sand flux increases with $h(x)$ from the foot of the dune to the crest and accordingly, $\mathcal{F}$ is an increasing function of the position on the dune $x$. As a result $u_{*}^{\prime}$ decreases with $x$ and therefore for gentle winds, erosion may occur only close to the crest and may flatten out the dune as proposed by Bagnold [1941]. Finally, assuming that the sand flux follows the same scaling as the saturated sand flux, $q_{s}$, the velocity of a shape-invariantly migrating two dimensional dune scales as

$$
c \sim \frac{\left(u_{*, \infty}-u_{*}^{\prime}\right) u_{*, \infty}^{2}}{H} .
$$

This is, however, only valid for scale-invariant shapes. This demonstrates that the scaling $c \sim 1 / H$ simply comes from: (1) the mass conservation, (2) the fact that the wind is turbulent and, (3) that the barchans are scale invariant. This scaling is in excellent agreement with field measurements, as long as dunes are assumed to be scale invariant, that is to say for large dunes compared to the minimal size. Finally, this scaling should also hold for three dimensional dunes since the same derivation can be done with width-averaged quantities.

[14] In the experiment the flow is turbulent $\left(R_{e} \sim 10^{4}\right)$. The barchans are large compared to the minimal size and are almost scale invariant so the sand flux can again be described by $\left(u_{*}-u_{*, c}\right) u_{*}^{2}$ [Julien, 1998]. Finally, the influence of the shape of the dune on the velocity field can also be described to the first order by equation (3) as long as the height of ripples is small compared to the water depth [Lagre, 2000]. Therefore the scaling equation (8) should be recovered in the case of the experiment.

\subsection{Influence of the Speed of the Plate}

[15] To study the influence of the speed of the flow, several experiment have been conducted with the initial acceleration, $\gamma$, kept constant to the value used for the motion of reference $\gamma_{0} \sim 1.8 \mathrm{~m} \mathrm{~s}^{-2}$, whereas $v$ has been tuned from $16.8 \mathrm{~cm} \mathrm{~s}^{-1}$ to almost $30 \mathrm{~cm} \mathrm{~s}^{-1}$. Each experiment starts with the same conical sand pile $(\sim 4 \mathrm{~cm}$ wide and made with a mass of $6.5 \mathrm{~g}$ of glass beads). Looking at the motion of the center of mass of the produced barchans allows to compute their mean velocity as function of the speed of the plate, $v$. In every case, after a short transient the conical sand pile transforms into a barchan that moves with a nearly constant speed (see inset of Figure 6). In Figure 6, one can see that $c \sim\left(v-v_{c}\right) v^{2}$ and accordingly $c \sim v^{3}$ for a not to weak "apparent wind". This is in keeping with our previous derivation assuming that the speed of the flow seen by the dune, $u$, is proportional to the speed of the plate, $v$ : a stronger flow increases the sediment transport rate on the surface of the dune and accordingly, the dune speed increases. Combining this result with the scaling $c \sim 1 / H$ found previously, the velocity of a barchan dune in a steady state can be written as

$$
c \sim \frac{\left(v-v_{c}\right) v^{2}}{H}
$$

Furthermore, it can be seen in Figure 6 that the shapes slightly changes with the wind strength. More precisely, the ratio $W / L$ seems to decrease when the wind strength 


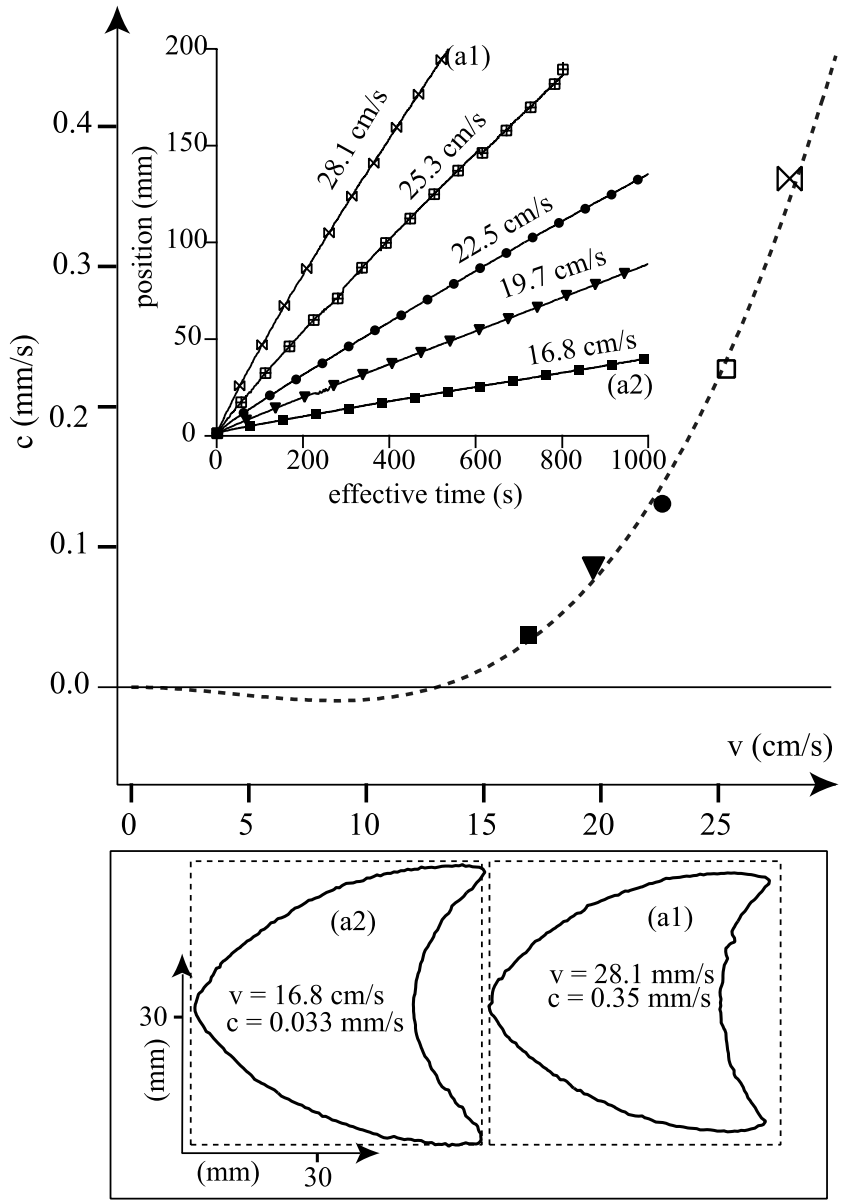

Figure 6. Influence of $v$ for $\gamma_{0}=1.87 \mathrm{~m} \mathrm{~s}^{-2}$. The inset shows the evolution of the position of the center of mass of barchans with the effective time of the motion, that is to say the time during which the motion of the plate produces a transport of sand $\left(t=t_{a}+t_{b}+t_{c}\right)$. In every experiment the barchans propagate in a quasi steady state regime, except during the formation of the barchan at the beginning of the experiment. The dashed line shows that the relation $c \sim$ $\left(v-v_{c}\right) v^{2}$ fits well with the experimental measurements with $v_{c}=12 \mathrm{~cm} / \mathrm{s}$. The influence of the velocity of the flow on the shapes is shown in the case of the fastest, $(a 1)$, and the slowest dune, $(a 2)$, for that series of experiments. It shows that the shape remains approximately the same, even if the fastest dune seems to have a lower aspect ratio $W / L$.

increases confirming the conjecture of Wippermann and Gross [1986].

\subsection{Unsteady Structures Formed for Extreme Values of the Acceleration of the Plate}

[16] The role of the initial acceleration of the plate is still to be clarified. So far there exists no general study of the effect of time-dependent flow on the transport rate of sand, though this is a very significant issue since the intensity of wind or water flow generally fluctuates with time in natural environments. However, it is possible to shed light on its role and its limitation in the presented experiment. Considering that the fluid is at rest initially, laminar boundary layer theory allows to estimate the evolution of the shear stress, $\tau$, in the thin laminar region close to the surface of the plate as

$$
\tau(t)=\rho v \sqrt{\frac{\nu t}{\pi t_{a}}}
$$

where $\rho$ is the water density and $\nu$ the viscosity. It appears that the main role of acceleration is to overcome the threshold of motion of grains. Reducing the time $t_{a}$ and therefore increasing the initial acceleration increases the shear-stress, and accordingly ease the incipient motion of grains. This is the very reason why this type of experiment is so efficient and gives very quick dynamics. With a constant flow, the boundary layer is fully developed and the shear stress near the ground is far less important. This is why experiments done in classic water flumes have longer timescale of evolution (typically several hours instead of typically 1 hour). Moreover, the acceleration process does not last for a long time and the mean transport is still governed mainly by the final speed of the plate as shown in the previous section (see Figure 6). In other words, the acceleration phase can be seen as an activation of the erosion process.

[17] However, significant changes are expected if $t_{a}$ becomes too short or too long, and accordingly if the initial acceleration $\gamma=v / t_{a}$ becomes too strong or too weak. Two experiments have been done with extreme values of $t_{a}$ and with the speed of the motion of reference, $v=28 \mathrm{~cm} \mathrm{~s}^{-1}$, kept constant. For a very short acceleration time $t_{a}=0.07 \mathrm{~s}$, the initial sand pile quickly gets the crescentic shape but never reaches a steady state regime as shown on Figure 7.
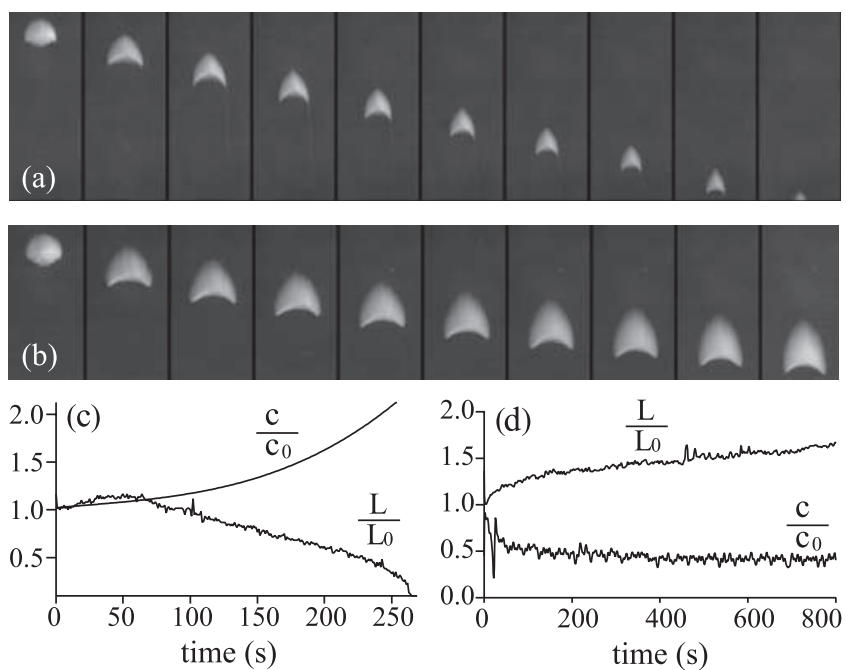

Figure 7. Unsteady subaqueous sand dunes created with extreme values of $\gamma$. There are 10 oscillations of the plate between two pictures. (a, c) Here $\gamma=4 \mathrm{~m} \mathrm{~s}^{-2}$. In comparison to the standard case (see Figure 3 ), the barchan propagates very quickly while shrinking. (b, d) Here $\gamma=$ $0.47 \mathrm{~m} \mathrm{~s}^{-2}$. In this situation the dune decelerates and flattens out. Figures $7 \mathrm{c}$ and $7 \mathrm{~d}$ show that in these peculiar conditions of acceleration the velocity scales as the inverse of the length rather than the inverse of the height. The speed of dunes changes while the shape and size of barchans are modified. 
On the contrary, the barchan dune accelerates up to a speed of $0.083 \mathrm{~cm} \mathrm{~s}^{-1}$. The shrinking of the dune indicates that the output sand flux is also increased. This is in agreement with the idea that the acceleration helps to set the grains into motion: for too high accelerations grains can be put into suspension, increasing dramatically the transport of sand. Strikingly, the speed of the dune still varies as the inverse of a typical dimension of the barchan. This may be explained by the fact that the barchan remains roughly scale invariant while shrinking and that the output sand flux is still small compared to the overall sand flux and therefore the mass conservation roughly gives $c \sim Q(\gamma, v) / H$. This suggests that in presence of an incoming sand flux, this experiment may produce barchans moving in a steady regime. Notice that for too violent acceleration the sand pile just flattens out because of mechanical vibrations. For this reason, it is not possible to decrease $t_{a}$ below $0.050 \mathrm{~s}$.

[18] On the contrary for $t_{a}=0.6 \mathrm{~s}$ the acceleration time is of the same order than the duration of the steady movement of the plate $\left(t_{b} \simeq 0.5 \mathrm{~s}\right)$. An initial conical sand pile transforms very slowly to a flattening and decelerating dune (see Figure 7). This figure further shows that the length and the speed evolves almost inversely proportionally. This is in keeping with the theoretical argument of Kroy [2002] and Momiji et al. [2002] who claim that the speed of a dune should depend on its length rather than on its height. Using the approximation $\mathcal{F} \sim H / L$, the increase of the sand flux at the crest is $q_{c}-q_{0} \sim H / L$ and therefore the speed of the dune scales as $c \sim 1 / L$. This effect was not observable in the case of the steady propagating dune since in that case the length, the width and the height are linearly dependent.

[19] Further studies are required to capture the influence of the acceleration process and to learn more about its importance. However, using an acceleration around the one used in the motion of reference always gives rise to welldefined barchans whose properties are closely related to those of aeolian barchans.

\section{Complex Dynamics of Barchans: New Insights}

[20] The experimental setup described in the previous section turns out to be a very simple and efficient way to reproduce the shape and dynamics of barchans but at much smaller scale and with shorter timescale of evolution. It is thus tempting to explore briefly two more complex issues: the influence of the wind directionality and the mechanism of collisions of barchans.

\subsection{Influence of Wind Direction}

[21] In the ideal picture of barchan dunes that has been sketched so far, the wind is supposed to be unidirectional. However, basic observations show that this is only the case on average [Pye and Tsoar, 1990; Cooke et al., 1993]. In deserts, the direction of the wind rotates during sand storms [Bagnold, 1941; Cooke et al. 1993]. In such events, the speed of the wind is very large and accordingly the erosion should lead to considerable change of the shape of dunes. On the other hand, the direction of the wind can also fluctuate, albeit more slowly, with the change of seasons for example. Moreover, under water large barchan dunes may be formed by asymmetric tidal current: the direction of the flow fluctuates every day. These variations of the direction of the flow have mainly two effects. The first is that the position of the crest may oscillate and therefore avalanches may develop in more than one direction, leading to a complex stratification inside the dune [Pye and Tsoar, 1990; Cooke et al., 1993; Le Bot and Trentesaux, 2004]. The second consequence is the remodeling of the external shape. This process takes more or less time depending on the size of the dune, and therefore fluctuations in the wind direction can lead to more or less deformed barchans. This is why wind fluctuations are often used as a fundamental reason to explain the asymmetry or the peculiar shape of some aeolian barchans in the field. However, hardly anything is known about the process of adaptation of a barchan to a change of the wind direction. Without pretending to explore this topic completely, it is exciting to use the previous setup to observe the deformation of a barchan with time when the direction suddenly changes.

[22] As in the previous section, starting from a conical sand pile, a subaqueous barchan forms and propagates. When the barchan reaches the center of the plate, the plate is rotated of the angle $\theta$. When the motion starts again, the barchan "mutates" in order to adapt to the new "wind" configuration, and the camera simply records the evolution of the shape. Figure 8 shows two different experiments with $\theta=90^{\circ}$ and $\theta=180^{\circ}$. Strikingly, the barchan dune adapts to the new wind direction supporting the "attractor" quality of the crescent morphology [Werner, 1995]. However, in both cases, while adapting the subaqueous barchan loses a small part of its mass, creating several small barchans. This very basic observation is of particular importance since it illustrates one possible way of nucleation for aeolian barchans. In particular, in the case of the $\theta=90^{\circ}$ rotation, the breaking of the horn seems to originate because one part of it is submitted to the external flow whereas the other part is shelter inside the recirculation bubble. Therefore one part of the horn moves and finally separates from the main body of the barchan. As every sand patch of sufficient size, it takes the shape of a barchan while migrating away from the upwind dune.

[23] Furthermore, this experiment can be used to investigate the importance of the directionality of the wind on the selection of dunes shapes. For example, if the timescale of fluctuations of the direction of the wind is shorter than the turnover time, and if the wind has no particular directionality, then no "barchan dune" can form but the initial sand pile should eventually transform into a star-like dune. Starting from a large sand pile ( $\sim 15 \mathrm{~cm}$ wide) in order to increase the turnover time of the future dune, and rotating the plate randomly allows us to create non migrating shapes with several arms. Such a subaqueous star dune is shown on Figure 9. The issue of the relation between the directionality of the wind and the shape of solitary dunes should be addressed quantitatively in the future.

\subsection{Binary Collisions: Toward Dune Interactions}

[24] Aside from the influence of the directionality of the wind, barchans can be more or less affected by their close neighbors. In particular, barchans of different sizes have different speeds, and therefore, collisions are likely to appear in any assembly of barchans. Under water, barchanoid ridges can be seen as a region with a very high density of barchans touching each other. In such a case, interactions 


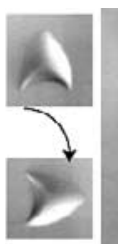

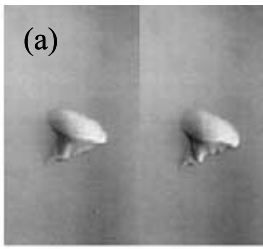

$t=1$ 2
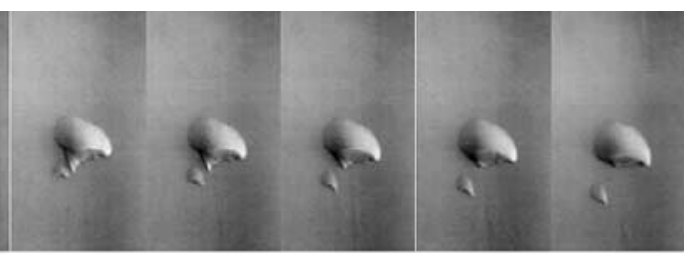

3

4

5

6

7
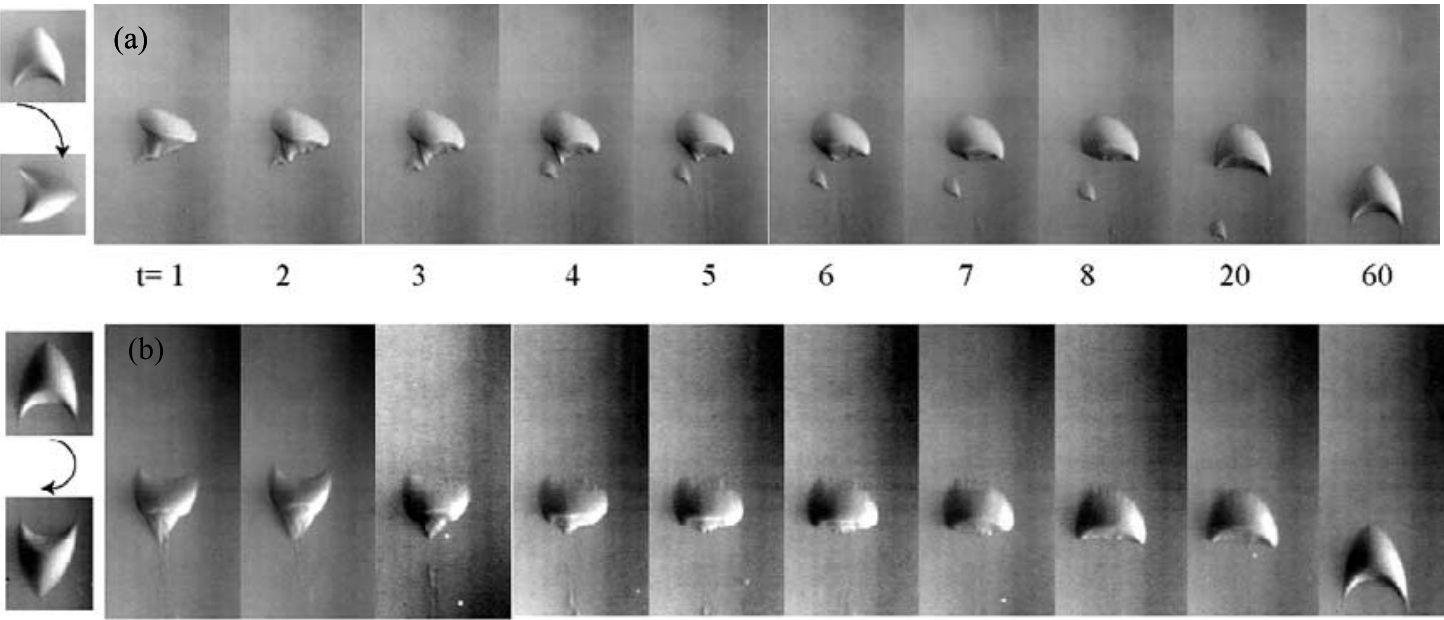

8

20

60

$$
\mathrm{t}=1 \quad 3 \quad 5 \quad 5
$$

9

11

13

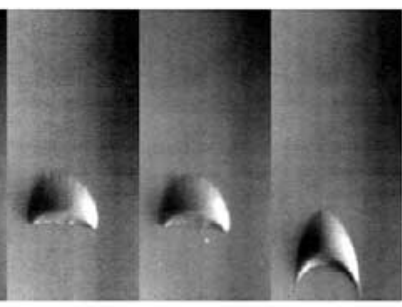

15

36

51

Figure 8. (a) Subaqueous barchan evolution after a rotation of $\theta=90^{\circ}$. The barchan dune $(5 \mathrm{~cm}$ wide) recovers its initial orientation after only a few strokes. The adaptation is done thanks to the destruction of one of the downwind horns, which separates from the dune and forms a tiny and fast subaqueous barchan. (b) Barchan evolution after a rotation of $\theta=180^{\circ}$. Again, the barchan adaptation takes only a few oscillations of the plate. However, the morphological evolution is completely different, since the barchan literally moves the other way around, its horns moving from the upwind side to the downwind side.

between barchans take place everywhere and are especially difficult to understand. However, it is possible to start by studying the simple case of a binary collisions. Using the experimental setup with two different conical sand piles allows us to observe easily a collision. The temporal evolution of such an event is shown on Figure 10. Strikingly the collision does not appear to be a simple coalescence process or a simple crossing [Schwämmle and Herrmann, 2003]. It rather appears to be an absorption/emission process, where the small impacting dune merges into the big one, while the latter emits one or several small barchans. The emission of small barchans seems to come from the change in the incoming sand flux (the excess sand arriving on the dune is directed preferentially on one horn) and the erosion due to the flow in the wake of the incoming dune. Notice that collisions may explain the formation of small barchans and should be involved in the size distribution of barchans in a corridor of dunes. This almost unknown and especially exciting issue can motivate future study on barchan dunes.

\section{Discussion and Conclusion}

[25] In this article, it has been shown that a simple experimental setup can be used to produce and manipulate subaqueous, propagating crescentic dunes. These underwater barchans have the same morphology and dynamics as aeolian barchan dunes. The close resemblance is theoretically supported by the existence of a unique characteristic lengthscale: the saturation length. This length, linked to the inertia of sand grains, scales as the ratio of the density of the sand and that of the fluid times the mean diameter of grains. The relevance of this length has been illustrated here by showing that aeolian and underwater barchans match once rescaled by this length. The use of this experiment is therefore a convenient way to explore many complex issues related to the dynamics of barchans. As a matter of fact, it is hardly feasible to achieve precise dynamical studies of barchans in the field because of the very slow evolution of their shape. On the contrary, in the experiment the typical timescale of evolution is just of about 1 hour.

[26] Therefore, using this experiment, it has been shown that the speed of steady subaqueous barchans scales as the cube of the velocity of the flow and as the inverse of one of

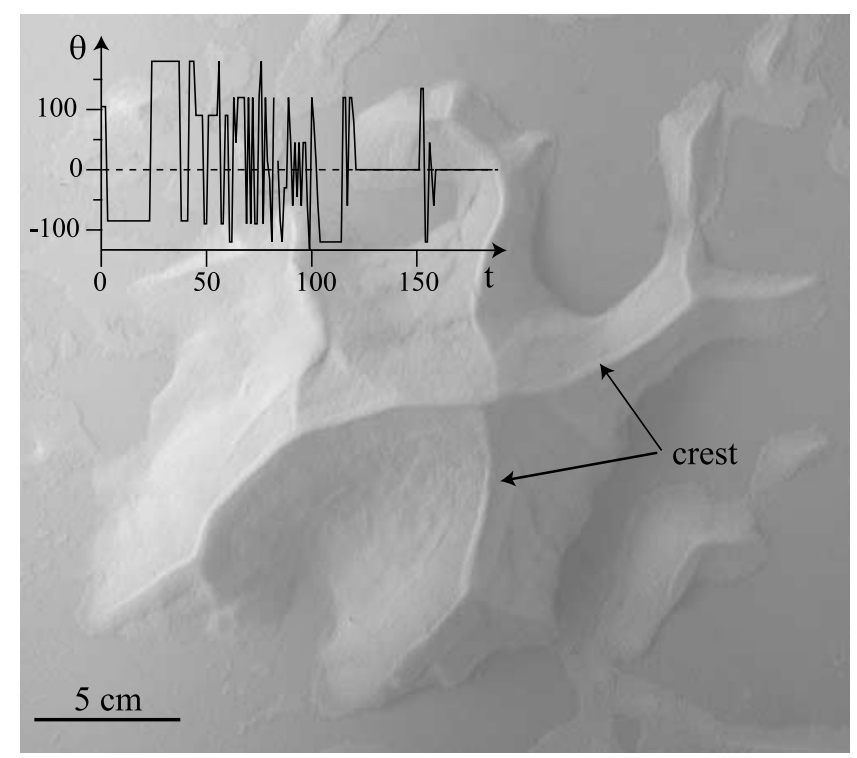

Figure 9. Influence of the directionality of the apparent wind. The randomized rotation of the plate leads to the formation of a star-like dune. This shows that if the typical timescale of evolution of the dune is much smaller than that of the fluctuations of direction of the wind, no propagating crescentic shape is recovered. 

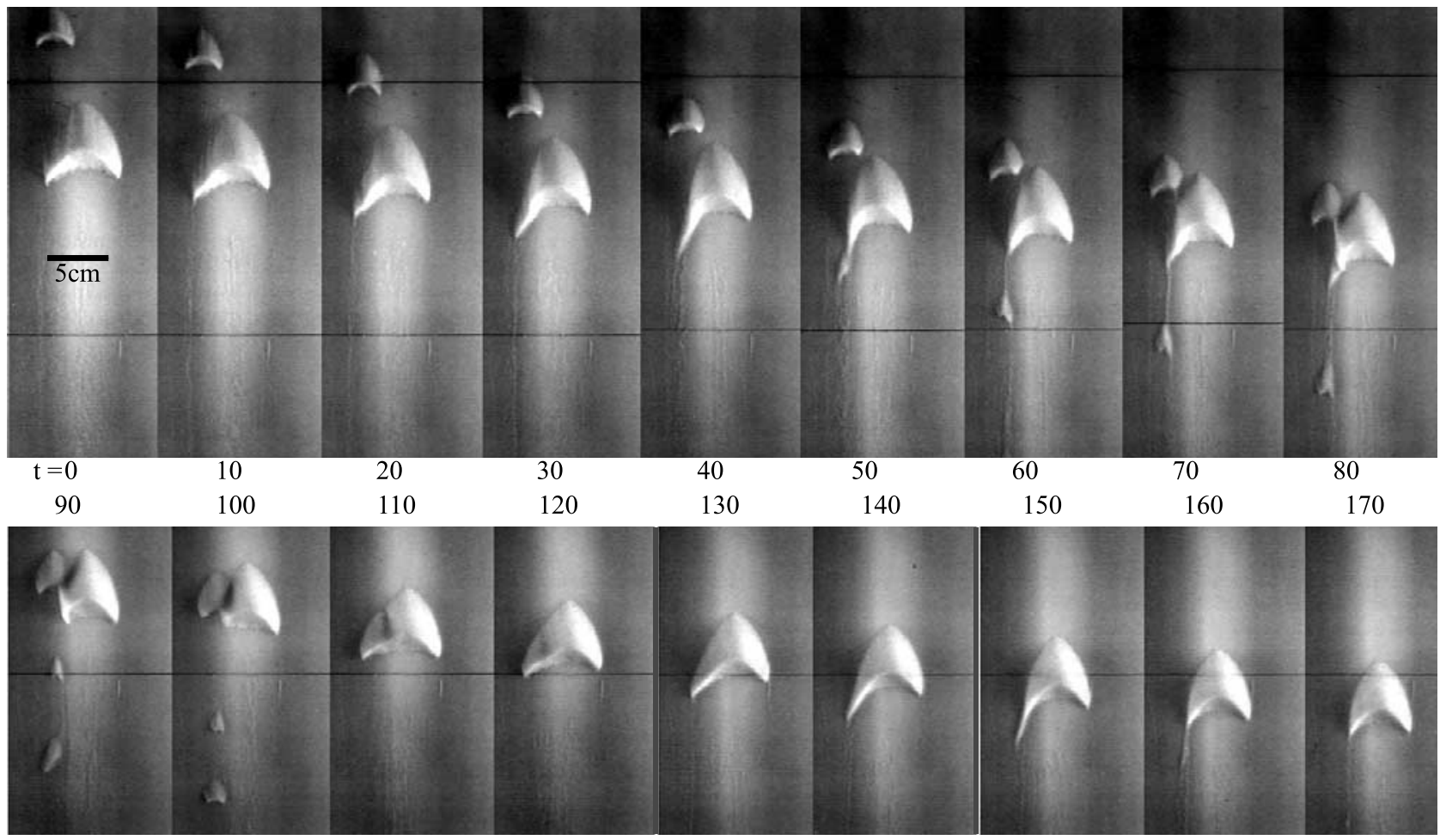

Figure 10. Mechanism of a binary collision. The smaller barchan is fast enough to enter in collision with the bigger one. This leads to a complex merging and eventually to the emission of small barchans while the big one readapts to a classic crescentic shape. There are 10 strokes of the plate between each picture.

their typical dimensions. The close relation between subaqueous barchans and aeolian dunes strongly suggests that aeolian barchans are moving in the same way. Finally, the experiment has been used to open the way to new issues. First we showed how a barchan reacts to a sudden and brutal change of the wind direction by emitting one or several small barchans and finally recovering its shape. We have further shown that decreasing the directionality of the wind naturally leads to star dune like shapes. The last qualitative study deals with collisions of barchans. It has been shown that a collision of two barchans is a complex process in which the two colliding barchans merge while one or several small barchans are emitted, and propagate far downwind. This experiment is of particular interest, since it is the first step in understanding an assembly of dunes as the interaction of many elementary shapes.

[27] From this experimental work, several relevant issues has to be outlined. First, the issue of the role of the acceleration process in the experiment needs further investigation. This can be inscribed in the more general framework of the study of the transport of sand by unsteady flow. This is a crucial topic, since unsteady flows are often observed in nature (wind bursts or turbulent fluctuations for example). Secondly, it would be interesting to look systematically at the effect of the directionality of the "wind" on the shape of solitary subaqueous dunes. This may lead to a better understanding of the mode of deformation of barchans. It would also allow us to observe dynamically the transition between barchans and longitudinal dunes or even star dunes. In relation to marine sandwaves issues, it would be very interesting to understand the link between the sand availability and the size of the barchan dunes created. This issue is also related to the question of the selection of size of barchans, and is of particular relevance both for the understanding of aeolian or underwater corridors of dunes. Following this remark, more systematic studies concerning the collision of barchans will be highly valuable to the understanding of the long time evolution of corridors of barchan dunes or even to the description of underwater barchanoid ridges. As a matter of fact, very recent work [Hersen et al., 2004] has proposed that the stability of corridors of barchan dune may be due to either the fluctuations of the wind direction or binary collisions of dunes, because during these events several small barchans are emitted. This assumption may be extended to the case of underwater ripple fields where interactions of dunes are more frequent.

[28] Acknowledgments. The author is grateful to C. Léonard, T. Bohr, S. Douady and F. Malloggi for support and discussions about the present work. Nothing could have been achieved without the technical knowledge of L. Quartier. Finally, many thanks are owed to the organizers and the attendants of the MARID 2004 conference. An A.C.I J. Ch. supported this work.

\section{References}

Allen, J. R. L. (1968), Current Ripples: Their Relation to Patterns of Water and Sediment Motion, Elsevier, New York.

Anderson, R. S., M. Sørensen, and B. B. Willetts (1991), A review of recent progress in our understanding of aeolian transport, Acta Mech., 1, Suppl., $1-19$.

Andreotti, B., P. Claudin, and S. Douady (2002), Selection of dune shapes and velocities: Part 1. Dynamics of sand, wind and barchans, Eur. Phys. J. $B, 38,341-352$. 
Bagnold, R. A. (1941), The Physics of Blown Sand and Desert Dunes, CRC Press, Boca Raton, Fla.

Best, J. L. (2004), The dynamics and morphology of river dunes: Synthesis and future research directions, in Marine Sancwave and River Dune Dynamics II: International Workshop Proceedings, edited by S. Hulscher, T. Garlan, and D. Idier, pp. 1-6, Univ. of Twente, Enschede, Netherlands.

Cooke, R., A. Warren, and A. Goudie (1993), Desert Geomorphology, UCL Press, London.

Dauchot, O., F. Léchenault, C. Gasquet, and F. Daviaud (2002), Barchan dunes in the lab, C. R. Mec., 330, 185-191.

Hansen, J. L., M. van Hecke, A. Haaning, C. Ellegaard, K. H. Andersen, T. Bohr, and T. Sams (2001), Instabilities in sand ripples, Nature, 410, 324

Hastenrath, S. L. (1967), The barchans of the arequipa region, southern Peru, Z. Geomorphol., 11, 300-331.

Hastenrath, S. L. (1987), The barchan dunes of southern Peru revisited, Z. Geomorphol., 31, 167-178.

Hersen, P., S. Douady, and B. Andreotti (2002), Relevant length scale for barchan dune, Phys. Rev. Lett., 89, 264301.

Hersen, P., K. H. Andersen, H. Elbelrhiti, B. Andreotti, P. Claudin, and S. Douady (2004), Corridors of barchan dunes: Stability and size selection, Phys. Rev. E, 69, 011304.

Julien, P. Y. (1998), Erosion and Sedimentation, Cambridge Univ. Press, New York.

Kroy, K. (2002), Minimal model for Aeolian sand dunes, Phys. Rev. E, 66, 031302 .

Lagre, P. Y. (2000), Erosion and sedimentation of a bump in fluvial flow, C. R. Acad. Sci., Ser. IIb, 328, 869-874.

Le Bot, S., and A. Trentesaux (2004), Architecture of very large submarine dunes influenced by tide and wind-generated processes (Dover Strait, northern France), in Proceedings of MARID 2004, 2nd International Workshop on Marine Sandwave and River Dune Dynamics, edited by
S. J. M. Hulscher, T. Garlan, and D. Idier, pp. 184-191, Univ. of Twente, Enschede, Netherlands.

Lettau, K., and H. H. Lettau (1969), Bulk transport of sand by the barchans of La Pampa La Hoja in southern Peru, Z. Geomorphol., 13, 182-195.

Mantz, P. A. (1978), Bedforms produced by fine, cohesionless, granular and flakey sediments under subcritical water flows, Sedimentology, 25, 83103.

Momiji, H., H. Nishimori, and S. Bishop (2002), On the shape and migration speed of a proto-dune, Earth Surf. Processes Landforms, 27, 1335-1338.

Pye, K., and H. Tsoar (1990), Aeolian Sand and sand dunes, CRC Press, Boca Raton, Fla.

Sauermann, G. (2001), Modeling of wind blown sand and desert dunes, Ph.D. thesis, Univ. Stuttgart, Stuttgart, Germany.

Sauermann, G., P. Rognon, A. Poliakov, and H. J. Herrmann (2000), The shape of the barchan dunes of southern Morocco, Geomorphology, 36, $47-62$.

Schwämmle, V., and H. J. Herrmann (2003), Solitary wave behavior of sand dunes, Nature, 426, 619 .

Valance, A. (2005), Formation of ripples over a sand bed submitted to a turbulent shear flow, Eur. Phys. J. B, 45, 433-442.

Valance, A., and V. Langlois (2005), Ripple formation over a sand bed submitted to a laminar shear flow, Eur. Phys. J. B, 43, 283-294.

Werner B. T. (1995), Eolian dunes: Computer simulations and attractor interpretation, Geology, 23((12)), 1107-1110.

Wippermann, F. K., and G. Gross (1986), The wind-induced shaping and migration of an isolated dune: A numerical experiment, Boundary Layer Meteorol., 36, 319-334.

P. Hersen, Laboratoire de Physique Statistique, Ecole Normale Supérieure, 24 rue Lhomond, 75005 Paris, France. (hersen@1ps.ens.fr) 